\title{
JAK2/Src Inhibitor NS-018
}

National Cancer Institute

\section{Source}

National Cancer Institute. JAK2/Src Inhibitor NS-018. NCI Thesaurus. Code C98109.

An orally bioavailable, small molecule inhibitor of Janus-associated kinase 2 (JAK2) and Srcfamily kinases, with potential antineoplastic activity. JAK2 inhibitor NS-018 competes with ATP for binding to JAK2 as well as the mutated form JAK2V617F, thereby inhibiting the activation of JAK2 and downstream molecules in the JAK2/STAT3 (signal transducer and activator of transcription 3) signaling pathway that plays an important role in normal development, particularly hematopoiesis. In addition, NS-018 inhibits the Src family tyrosine kinases. This eventually leads to the induction of tumor cell apoptosis. JAK2 is the most common mutated gene in bcr-abl-neg ative myeloproliferative disorders (MPDs); JAK2V617F is a constitutively activated kinase that activates the JAK/STAT signaling pathway and dysregulates cell growth and function, and its expression transforms hematopoietic cells to cytokine-independent growth. 